\title{
Adaptation of Sporocytophaga myxococcoides to Sugars
}

\author{
By ANTJE KAARS SIJPESTEIJN AND G. FAHRAEUS \\ Laboratory for Microbiology, Technical University, Delft, Holland
}

SUMMARY: Strains of Sporocytophaga myxococcoides were cultivated in a mineral nutrient medium with glucose. In media containing $0 \cdot 1 \%$ glucose sterilized by Seitzfiltration growth readily occurred, whereas in corresponding media with 0.5 and $1.0 \%$ glucose there was a pronounced lag in development that sometimes lasted for several weeks.

The duration of the lag period appeared to depend on the number of cells in the inoculum, and could be diminished by incubation at $25^{\circ}$ instead of $30^{\circ}$, although the latter temperature is nearer to the optimum temperature for growth. Apparently adaptation to the high glucose concentration takes place during the lag period.

Cells adapted to high glucose concentrations grow readily in media with the same concentration of mannose, whereas non-adapted cultures do not grow in these media, or only after a long lag period. None of the strains investigated showed any development in media in which either fructose or xylose was the sole source of carbon; nevertheless, these sugars did not inhibit, or only slightly, inhibited cellulose decomposition.

Stanier's observation, that the development of $S p$. myxococcoides is inhibited when the glucose is autoclaved in the nutrient medium, was confirmed. If, however, due care be taken to avoid an alkaline reaction, glucose autoclaved either separately or in the medium sustains growth in the same way as does glucose sterilized by filtration.

For several types of cellulose-decomposing micro-organisms the ability to hydrolyse cellulose to the constituent sugars, either cellobiose or glucose, has been demonstrated. This, as well as the fact that at least some of these organisms have been found to grow on glucose as a sole source of carbon, has led to the general conception that cellulose breakdown by micro-organisms proceeds in the following two steps: (1) hydrolysis to glucose, succeeded by (2) oxidation or fermentation of this sugar to the final metabolic products. However, until recently cellulose-decomposing soil bacteria of the Cytophaga group seemed to be an exception to this rule. In spite of several attempts nobody had been successful in demonstrating either cellulase activity or growth on glucose. Moreover, the fact that even low concentrations $(0 \cdot 1-0 \cdot 2 \%)$ of reducing sugars, glucose included, retarded or even completely inhibited cellulose decomposition, suggested a toxic action of this sugar towards Cytophaga spp. As the older literature on this subject is discussed extensively in the papers of Stanier (1942 $b$ ) and Fahraeus (1947) we shall not enter into details here.

Fåhraeus (1941) attacked this problem from the quantitative side. With the aid of glucose and cellulose determinations he showed that glucose, added in low concentrations $(0.05 \%)$ to the cellulose medium, was rapidly consumed by both strains studied, and further that the observed delay in the cellulose decomposition was due to a preferential attack on the glucose. However, when using higher glucose concentrations he observed a lag in the development during which neither cellulose nor glucose was consumed. For the strain of 
'Cyt. globulosa' received from Stapp, this occurred even in $0.1 \%$ glucose, but Fåhraeus's own strain ' 3 ' was less sensitive. After the initial growth inhibition, which lasted for about 10 days, glucose was consumed first, and then cellulose.

The demonstration that Cytophaga spp. were able to use glucose made it plausible that like other cellulose-decomposing organisms, they would hydrolyse this polysaccharide previous to oxidation. Further evidence for a hydrolytic breakdown was given later (Fåhraeus, 1944, 1946, 1947).

Though the retardation of cellulose decomposition effected by low glucose concentrations had been satisfactorily explained by the fact that glucose is consumed preferentially, the phenomenon of the delay of both glucose and cellulose consumption caused by higher glucose concentrations required further investigation.

In 1942 Stanier published two papers on the Cytophaga group, dealing also with its glucose metabolism. At that time, owing to war conditions, Stanier was not yet acquainted with Fåhraeus's results. The important discovery was made by Stanier that glucose not sterilized by heating, but by Seitz-filtration, was, at least in a liquid medium, an excellent source of carbon for his Sporocytophaga and Cytophaga strains. This appeared to be true for glucose concentrations up to $2 \%$, maximal growth occurring after 5 days in all concentrations, in tubes without cellulose. Stanier states in this connexion: 'Furthermore, the glucose sterilized by filtration exerted no "toxic effect" on the growth in the presence of cellulose. With the highest concentrations tested there was, it is true, a decrease in cellulose decomposition, but at the same time abundant growth occurred in the surrounding liquid medium. This partial inhibition of cellulose decomposition is probably due to preferential utilization of the monosaccharide.' One should compare these results with Stanier's experiments with glucose, autoclaved in the nutrient medium, where $0.1 \%$ or less of glucose in the cellulose medium supported maximal growth after 6 days, but $0.5-2 \%$ even after 20 days did not support any growth. Stanier suggested that the toxic effect must be a result of the formation of harmful decomposition products from the glucose when heated in the phosphate-containing medium. However, he did not describe experiments with glucose autoclaved separately in distilled water.

Fåhraeus (1947) made it clear that throughout his earlier work he had used glucose sterilized in this way, and that he had nevertheless observed inhibition. From this, one might conclude that heating glucose in distilled water also gives rise to inhibiting substances. However, this need not necessarily be true, for in Fåhraeus's experiments the glucose was toxic irrespective of any heating, since, contrary to Stanier, he observed inhibition also with Seitz-filtered glucose (cf. Fåhraeus 1947, p. 53). The same results were obtained with different samples of glucose, purified glucose, and glucose prepared by hydrolysis of lichenin by means of enzyme preparations of Cytophaga spp. These experiments were all performed with Stapp's strain of 'Cyt. globulosa'. Thus there remained a marked contrast between the results of Stanier and Fåhraeus, which still had to be explained. 


\section{Description of strains}

Five strains were available. As all formed spherical microcysts and resembled Sporocytophaga myxococcoides Hutchinson \& Clayton emend. Krzemieniewska (Stanier, 1940), in most other features, they may all be considered to belong to this species.

Two of the strains had been used by Fåhraeus throughout his earlicr experiments (1941, 1944, 1946, 1947), i.e. Cytophaga ' 3 ' and 'Cytophaga globulosa'. The latter strain was isolated and described by Stapp \& Bortels (1934).

The three other strains had been isolated in 1942 from garden soil by A. Kaars Sijpesteijn. These strains are designated $\mathrm{H}, \mathrm{W}$ and $\mathrm{D}$ (Pl. 1, figs. 1-3). After preliminary purification in liquid media they were isolated in the following different ways. Strain $\mathbf{H}$ was obtained in pure culture by application of the dilution method, strain $\mathrm{W}$ by the cellulose agar technique, and strain $\mathrm{D}$ by 'pasteurization' for $10 \mathrm{~min}$. at $70^{\circ}$. Microscopic examination of the cultures indicated the absence of any contaminants, and streaking on ordinary nutrient media did not give any growth, so that the purity of the cultures seemed guaranteed.

The length of the rods of these three strains is approximately the same, about $3-5 \cdot 5 \mu$, and the diameter of their microcysts is approximately $1 \cdot 6 \mu$. Strain $\mathrm{D}$ produces a pale yellow pigmentation and does not produce much slime, whereas strains $\mathrm{H}$ and $\mathrm{W}$ cause a deep yellow orange coloration of the paper accompanied with more abundant slime production.

The first author (A. K. S) had established in 1942 that these strains were able to grow, though badly, in media with, as the sole source of carbon, $0 \cdot 1 \%$ of glucose which had been sterilized by autoclaving in the complete medium.

In view of Stanier's results these experiments were repeated in 1946 shortly after receipt of his publication. It then appeared that it was indeed possible to obtain good growth in media containing the said low glucose concentration also when heat sterilization was applied. Hitherto it had been found necessary either to autoclave the glucose separately in distilled water, or to keep the $\mathrm{pH}$ of the medium to be sterilized at a value not exceeding $7 \cdot 0$. However, when the glucose was autoclaved in an even slightly alkaline medium, no development took place. The behaviour of the three strains in media with higher glucose concentrations had not been tested before the present work was undertaken.

\section{Methods}

For the experiments culture tubes containing $9 \mathrm{ml}$. of a mineral medium with the composition given below, and various concentrations of glucose, were used. The glucose was added as a concentrated solution in distilled water which was separately sterilized, either by Seitz-filtration or by autoclaving for $15 \mathrm{~min}$. at $110^{\circ}$. The figures given below refer to the final concentrations after addition of glucose to the basal mineral medium. (The glucose used was 'Analar', British Drug Houses Ltd.) 
Sometimes parallel tubes were set up both with and without the addition of cellulose (as strips of filter paper No. 597, Schleicher \& Schüll). As, however, growth was often poorer in the presence of filter-paper than in its absence, the paper strips were as a rule left out in the experiments reported here. The unfavourable effect of the filter-paper may be due to harmful substances in it (Fåhraeus, 1947).

The media indicated in Table 1 were used; they proved to be equally satisfactory.

Table 1

\begin{tabular}{ll}
\multicolumn{1}{c}{ Medium Dubos } & (g.) \\
$\mathrm{NaNO}_{3}$ & $0 \cdot 05$ \\
$\mathrm{~K}_{2} \mathrm{HPO}_{4}$ & $\mathbf{0 . 1}$ \\
$\mathrm{MgSO}_{4} \cdot 7 \mathrm{H}_{2} \mathrm{O}$ & $\mathbf{0 . 0 5}$ \\
$\mathrm{KCl}$ & $\mathbf{0 . 0 5}$ \\
$\mathrm{FeSO}_{4} .7 \mathrm{H}_{2} \mathrm{O}$ & $\mathbf{0 . 0 0 1}$
\end{tabular}

\begin{tabular}{|c|c|}
\hline Medium $\mathrm{g}$ & (g.) \\
\hline $\mathrm{NaNO}_{3}$ & $0 \cdot 05$ \\
\hline $\mathrm{K}_{2} \mathrm{HPO}_{4}$ & $0 \cdot 1$ \\
\hline $\mathrm{MgSO}_{4} \cdot 7 \mathrm{H}_{2} \mathrm{O}$ & 0.05 \\
\hline $\mathrm{KCl}$ & 0.05 \\
\hline $\mathrm{CaCl}_{2} \cdot 6 \mathrm{H}_{2} \mathrm{O}$ & 0.02 \\
\hline $\mathrm{FeCl}_{3} \cdot 6 \mathrm{H}_{2} \mathrm{O}$ & 0.002 \\
\hline $\mathrm{MnSO}_{4} \cdot 4 \mathrm{H}_{2} \mathrm{O}$ & 0.0002 \\
\hline
\end{tabular}

The $\mathrm{pH}$ was adjusted to give a value of $7 \cdot 2$ after autoclaving. All cultures were incubated at $30^{\circ}$, unless otherwise stated.

The tubes were inoculated with a loopful up to a few drops of a suspension either of attacked cellulose fibres taken from a young culture on filter-paper, or of the bacteria present in a previous culture in a sugar medium.

\section{Results}

Growth in liquid glucose-containing media

First the behaviour of four strains towards different concentrations of glucose autoclaved separately in distilled water was studied.

Table 2. Period necessary for obtaining visible growth of different Sporocytophaga strains in a medium with glucose autoclaved separately

Four parallel tubes: 2 without, 2 with filter-paper.

\begin{tabular}{|c|c|c|c|c|c|}
\hline & & & conce & $(\%)$ & \\
\hline & $0 \cdot 0$ & 0.05 & $0 \cdot 1$ & $0 \cdot 2$ & 0.5 \\
\hline & & & eriod & & \\
\hline 'C. globulosa' & $2-3$ & 3 & $4-5$ & 6-9 (i) & $9-14(i)$ \\
\hline 3 & 2 & $\mathbf{2}$ & 3 & 5 & 6 \\
\hline $\mathbf{H}$ & $3-4$ & $2-3$ & $2-5$ & 5 & 6 \\
\hline D & 2 & 2 & 4 & 5 & $5-6$ \\
\hline
\end{tabular}

As shown in Table 2 the results were essentially the same for all strains, in that in all cases the development was markedly retarded above a certain glucose concentration. The results confirm those obtained previously (Fåhraeus, 1941, 1947). 'Cytophaga globulosa' seemed to be the most sensitive strain, but 
it also finally grew in the highest concentration, although somewhat irregularly. The slightly better growth of this strain than in earlier experiments (cf. Fåhraeus, 1947, p. 54) may be due to the use of a different medium. The medium used in the experiments reported here contained nitrate as a source of nitrogen, while most earlier experiments were carried out with ammonia as a nitrogen source. There were also certain other differences, but the decomposition of cellulose in the absence of glucose was unaffected by this change in the composition of the medium.

Subsequently, the growth of ' $C$. globulosa' and strain $\mathbf{H}$ in various concentrations of filtered glucose was compared with that in a medium with glucose autoclaved separately. A Jena glass filter (G 5 on 3) was used for the filtration. The results are summarized in Table $\mathbf{3}$.

Table 3. Period necessary for obtaining visible growth of two Sporocytophaga strains in media with filtered or separately autoclaved glucose

\begin{tabular}{|c|c|c|c|c|c|c|}
\hline & \multicolumn{5}{|c|}{ Substrate } \\
\hline & & \multirow[b]{2}{*}{$\begin{array}{l}\text { Filter- } \\
\text { paper }\end{array}$} & \multicolumn{4}{|c|}{ Glucose $(\%)$} \\
\hline & & & $\overparen{0.05}$ & $0 \cdot 1$ & 0.2 & 0.5 \\
\hline Strain & $\begin{array}{c}\text { Treatment of } \\
\text { glucose }\end{array}$ & \multicolumn{5}{|c|}{ Period (days) } \\
\hline 'C. globulosa' & $\begin{array}{l}\text { Filtered } \\
\text { Autoclaved }\end{array}$ & $2-3$ & $\begin{array}{c}3-4 \\
5\end{array}$ & $\begin{array}{l}6-9 \\
6-9\end{array}$ & $\begin{array}{l}9(i) \\
-\end{array}$ & $\begin{array}{l}- \\
-\end{array}$ \\
\hline $\mathbf{H}$ & $\begin{array}{l}\text { Filtered } \\
\text { Autoclaved }\end{array}$ & 2 & $\begin{array}{c}2-3 \\
2\end{array}$ & $\begin{array}{c}5 \\
4-6\end{array}$ & $\begin{array}{c}5 \\
\mathbf{5}(i)\end{array}$ & $\begin{array}{l}6 \\
6(i)\end{array}$ \\
\hline
\end{tabular}

From these results it is quite clear that, at least under the conditions of the experiment, the filtered glucose also was inhibitory, in agreement with previous results (Fåhraeus, 1947).

In other experiments it was found that strains $D$ and $W$ showed the same inhibition by glucose, independently of its pretreatment. An experiment in flasks with a shallow layer of liquid in order to secure good aeration, did not lead to a diminution of the lag phase in glucose media.

An experiment was made with strain $\mathbf{H}$ to investigate whether cultivation at $25^{\circ}$ would allow better growth in different concentrations of Seitz-filtered glucose than at $30^{\circ}$. Inoculation was restricted to one loopful of a suspension of attacked filter-paper. Within 3-7 days growth had begun in all tubes with filter-paper only, and in those with $0.1 \%$ glucose, whilst in tubes with 0.5 and $\mathbf{1 . 0} \%$ glucose no turbidity could be observed. However, after 16 days, growth became visible in the first of these tubes; within the next few days development started in more tubes. Thus $\mathbf{2 4}$ days after inoculation development was apparent in four out of six tubes with 0.5 and $1.0 \%$ glucose incubated at $25^{\circ}$ and in three out of fourteen tubes incubated at $30^{\circ}$. Thirty days after inoculation growth started in one more of the latter tubes. The other tubes did not show visible growth up to the time the experiment was discontinued, at 41 days. Once growth had become apparent, it was abundant within a few days. 
These results show that in order to start growth in 0.5 and $1.0 \%$ glucose an incubation temperature of $25^{\circ}$ is more favourable than $30^{\circ}$, although development in cellulose media is much more rapid at the latter temperature. As a rule it takes 5-6 days before growth becomes visible at $25^{\circ}$ as compared with 3 days at $30^{\circ}$. These results were repeatedly confirmed; Table 4 summarizes

Table 4. Effect of incubation temperature on time needed for visible growth of Sporocytophaga strain $\boldsymbol{H}$ in filtered glucose

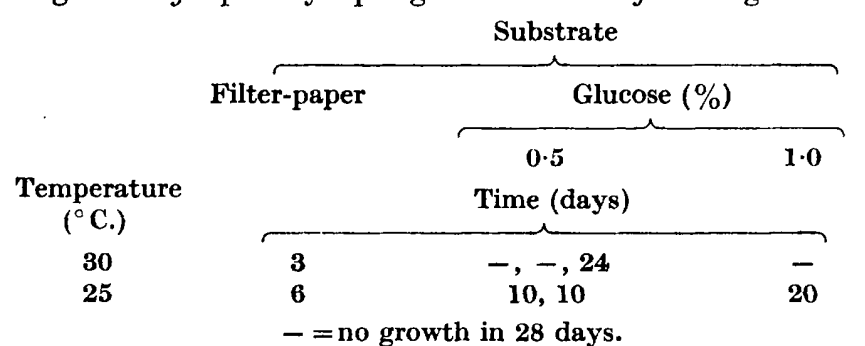

one of these experiments. Here again good growth in 0.5 and $1.0 \%$ glucose occurred only after a long delay. Obviously, in those tubes in which growth finally took place the cells of the inoculum were alive, but unable to grow during a lag phase which lasted for many days. We therefore conclude that during this period adaptation to the high glucose concentration takes place, as previously suggested (Fåhraeus, 1941, 1947). That in the experiments of Table 4 the lag was so much longer than in those summarized in Tables 2 and 3 may be ascribed to differences in strength of the inoculum. Earlier observations (Fåhraeus, 1947) support this view, which was further confirmed in an experiment in which tubes with $1.0 \%$ glucose were very heavily inoculated with attacked filter-paper taken from a culture with cellulose only; then growth usually was visible even after only 5 days at $30^{\circ}$. However, this experiment did not exclude the possibility that growth factors were introduced into the medium with the heavy inoculum and were really responsible for the observed shortening of the lag. But the experiment recorded in Table 5 appears to rule out this possibility.

Table 5. Number of days necessary for obtaining visible growth of 'Cytophaga globulosa' in glucose media on addition of: (1) a thick suspension of living cells; (2) the same suspension boiled; (3) the same after Seitz filtration

2,3 and control inoculated with a normal amount of bacteria.

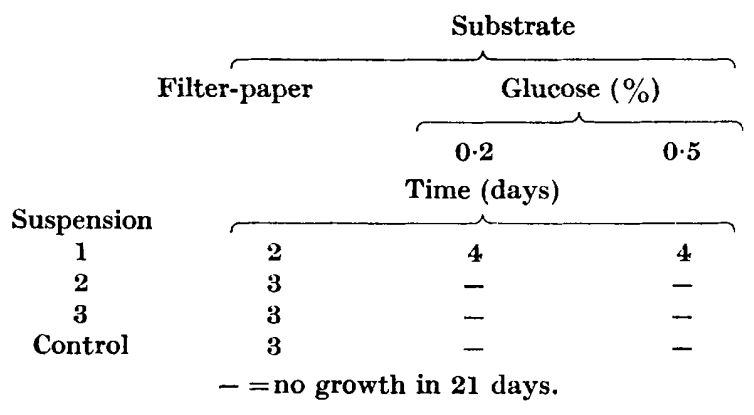


If the time required for adaptation is indeed responsible for the long lag period observed after inoculating from a cellulose culture, it would be expected that subculturing from a culture already grown in a glucose medium would not show any growth retardation.

Both for strain $\mathbf{H}$ and for strain $\mathbf{W}$ it was found that on continued transfer in media with the same high glucose concentration $(0.5$ or $1.0 \%)$ the first growth was already manifest after 3 days' incubation at $30^{\circ}$. This period is considerably shorter even than that observed in experiments in which very heavy inocula from cellulose cultures were used.

Besides adaptation to high glucose concentrations as such, it appeared that adaptation to a particular concentration of the sugar had taken place. In some experiments at least, in which tubes with $0.1,0.5$ and $1.0 \%$ glucose were inoculated from a young culture in a medium with $1.0 \%$ glucose, it was obvious that growth occurred much more readily in the tube with the corresponding sugar concentration, i.e. $1 \cdot 0 \%$.

Although the lag of cellulose-grown bacteria in media with high concentrations of glucose is considerably shorter at $25^{\circ}$ than at $30^{\circ}$, for continuous growth in glucose media $30^{\circ}$ is a more favourable temperature than $25^{\circ}$. This difference between the optimal temperatures for adaptation and for growth was established for all the strains tested, viz., H, W and D.

Cultures of Sporocytophaga growing in liquid glucose-containing media show some remarkable features. The first evidence of growth is nearly always the appearance of small yellowish granules at the surface of the sediment. One day later the liquid has become turbid, and often small floccules appear on the glass wall. Whereas filter-paper is practically only attacked near the surface of the medium, growth in glucose media is not restricted to the surface. Sometimes the turbidity is uniform throughout the medium; in many tubes, however, growth is granular and uneven, and both on the glass wall and in the liquid there occur small colourless or yellowish floccules, as described by Stanier $(1942 b)$. In old cultures there is always a thick layer of bacteria on the bottom of the tube, and the supernatant liquid becomes more or less clear.

The aerophilic character of the cells is clearly demonstrated by the heavy growth that occurs when the bacteria succeed in maintaining themselves on the surface of the medium. Pl. 1, fig. 4 demonstrates how under these conditions a thick pellicle is formed at the surface; when filter-paper is present this occurs more readily.

The larger floccules sometimes present on the glass walls were about $1 \mathrm{~mm}$. diameter and could easily be picked from the medium, and examined microscopically on a hollow-ground slide. After crushing, the material appears to be composed of both rods and microcysts. These colonies resemble the 'stars' described by Stapp \& Bortels for growth on a cellulose medium. In our case, however, the dimensions were much larger.

Pl. 1, fig. 5 shows bacteria obtained from a liquid medium containing $1 \%$ glucose. Contrary to its state in cells from cultures of the same age grown on cellulose, the chromatin is not evenly dispersed in the cells, but is localized; 
dividing rods, and the transition forms between rod and microcyst, are very frequent. The rods are somewhat inflated, and the microcysts are larger than in normal cultures. As these differences might be due to suboptimal conditions in the liquid medium owing to insufficient air supply, cells grown in the yellow ring at the meniscus of the water surface were also examined. They were however very similar, though less inflated. Also in bacteria grown in a liquid medium with $0.1 \%$ glucose the localization of the chromatinic material was rather conspicuous, but the cells were not inflated.

\section{Growth on glucose agar}

Since growth was good in a liquid medium with relatively high glucose concentrations, it was attractive to investigate the behaviour of the strains on nutrient agar containing filtered glucose. Stanier remarked that $S p$. myxococcoides developed scantily, if at all, on mineral agar with $1.0 \%$ glucose, and that development on plates was regular only when the sugar concentration did not exceed $0 \cdot 2 \%$. He suggested that the addition of glucose to the melted agar at $50^{\circ}$, just before pouring the plates, might cause sufficient decomposition of the sugar to affect adversely the subsequent development of the bacteria. Harmsen (1946) describes the formation of distinct colonies of strains of Sporocytophaga spp. on cellulose agar containing $0 \cdot 1 \%$ or $0.25 \%$ glucose.

In our attempts to cultivate Sporocytophaga on glucose agar we started from both cellulose and glucose cultures. We prepared plates of the same composition as Dubos's nutrient medium (Table 1 ) with $1.5 \%$ agar that had been carefully washed with distilled water for several days. The agar was cooled to $37^{\circ}$, mixed with a Seitz-filtered glucose solution at the same temperature, and the plates poured immediately.

In several experiments strain $\mathbf{H}$ grown in a $1 \%$ glucose medium was streaked on plates containing $1 \%$ glucose. Many colonies were seen after 5 days at $30^{\circ}$. After 12 days the largest colonies had a diameter of 2-3 mm. (Pl. 1, fig. 6). They were yellow-orange in colour, very slimy, and in the centres of the colonies darker parts of a peculiar shape were visible, all colonies being of the same character.

In another experiment, with the same strain, streaks were made from a heavy suspension of decomposed filter-paper on to 0.1 and $1 \%$ glucose agar. On the latter no growth occurred, but on $0.1 \%$ glucose agar a great many colonies were seen after 5 days. The colonies were rather faint in colour and less thick and slimy than those on $1 \%$ glucose described earlier. In this case too all colonies were of the same type (cf. Pl. 1, fig. 7).

In several other experiments it appeared that growth occurred more readily when the glucose plates were inoculated from cultures containing the corresponding concentration of glucose, once more suggesting that adaptation to the given concentration of the sugar had taken place.

It should be stressed that development on glucose agar was not always successful; in several cases growth failed completely notwithstanding that under the same conditions in other instances good growth had been obtained. We have no explanation for this. 
The microscopical appearance of bacteria grown on $0 \cdot 1 \%$ glucose agar can be seen in Pl. 1 fig. 8.

From strain $\mathrm{W}$ grown in $\mathbf{0} \cdot \mathbf{1}$ and in $\mathbf{1} \%$ glucose media, streaks were made on 0.1 and in $1 \%$ glucose agar. In addition, transfers were made from a heavy suspension of decomposed filter-paper to plates with both sugar concentrations. With the exception of the plates inoculated from the cellulose culture, in all cases colonies developed after 7 days' incubation at $30^{\circ}$. On these plates two types of colonies could be distinguished (cf. Stanier, 1942b), one of which might be called a typical rough form, the other a more or less smooth form. The rods from the smooth colonies were rather long and swollen, those of the rough colonies giving a more normal picture. A cellulose medium inoculated from a rough colony supported a more rapid growth than a similar medium inoculated from a smooth colony.

In a second experiment with the same strain streaks were made from a heavy suspension of attacked filter-paper on 0.1 and $1 \%$ glucose agar. On the latter, no growth occurred, but on $0.1 \%$ glucose agar many colonies were visible after 5 days incubation. Here too, rough (cf. Pl. 1, fig. 9) and smooth colonies could be distinguished, the smooth being somewhat smaller than in the first experiment.

\section{Growth on mannose, fructose and xylose}

Stanier's $S p$. myxococcoides did not attack mannose, whereas the two strains studied by Fåhraeus (1947) both did so. The remaining strains $\mathrm{H}, \mathrm{W}$ and D were now tested in this respect, and appeared to grow well on filtered mannose $(0 \cdot 1,0.5,1.0 \%)$. Nevertheless, here too there was a lag of about the same duration as in the experiments with glucose.

Cultures grown in $1 \%$ glucose medium were examined to see whether they would develop without delay in a medium containing the same concentration of mannose. Transfers were made from young cultures of strains $\mathbf{H}$ and $\mathrm{W}$ grown in $1 \%$ glucose to nutrient media with $1 \%$ mannose and with $1 \%$ glucose; the tubes were incubated at $30^{\circ}$. Growth was visible after 3-4 days in both media.

Experiments were carried out to investigate the suitability of fructose and xylose as a substrate for the strains $\mathrm{H}, \mathrm{W}$ and $\mathrm{D}$. The strains of Sporocytophaga spp. investigated by Stanier (1942b) and by Fåhraeus (1947) did not grow on any of these sugars. However, since xylan is consumed by several strains of $S p$. myxococcoides (Fuller \& Norman, 1942, 1943) these strains might grow on xylose. Tubes containing $1 \%$ filtered fructose or xylose were inoculated with cultures of the strains $\mathbf{H}, \mathbf{W}$ and $\mathrm{D}$ grown on $1 \%$ glucose. No growth occurred even after 23 days incubation. Control tubes containing $1 \%$ glucose inoculated at the same time showed visible growth already after 3-4 days. This experiment was repeated several times with the same negative results. Hence it is probable that for the strains tested fructose and xylose cannot act as a substrate.

Further experiments supported this view. Tubes of nutrient media provided with a strip of filter-paper and tubes which contained in addition $1 \%$ fructose 
or xylose were inoculated with a heavy suspension of attacked filter-paper. In the tubes without sugar the attack on the filter-paper was manifest after 2 days incubation; after 3 more days the strips disintegrated at the surface of the medium. In the tubes containing fructose or xylose a coloration of the filterpaper became visible after 2-4 days, and 3-5 days later here too the paper was disintegrated at the surface. The nutrient medium remained perfectly clear in all cases. These observations seem to indicate that neither fructose nor xylose had been consumed; the disintegration of the filter-paper was, however, slightly delayed by the presence of these sugars. The three strains $\mathbf{H}, \mathbf{W}$ and $\mathrm{D}$ gave similar results.

In another experiment we tested whether the strains could be adapted to growth on fructose or xylose; small pieces of filter-paper that had been attacked in a medium containing $1 \%$ fructose or $1 \%$ xylose failed to cause turbidity after transfer to nutrient media with the same concentrations of these sugars, whereas turbidity was visible after 4 days in analogous cultures in glucose media.

\section{Growth on glucose autoclaved in the nutrient medium}

Glucose in culture media is liable to decomposition by heat treatment, the more so in a slightly alkaline medium. The intensity of the yellowish tinge formed in glucose-containing media during autoclaving seems to run parallel to their inhibiting action upon certain micro-organisms. In earlier, unpublished investigations, one of us (A. K. S.), however, observed that $0 \cdot 1 \%$ glucose could be autoclaved in the nutrient medium without any unfavourable effect on the growth of Sporocytophaga, if the $\mathrm{pH}$ was not allowed to exceed $7 \cdot 0$ during the heating. Growth on such media was as good as in media containing $0 \cdot 1 \%$ glucose sterilized either by Seitz-filtration, or by heating in distilled water. When, however, the $\mathrm{pH}$ was $\mathbf{7 \cdot 2}$ or higher during autoclaving a marked inhibition of growth usually occurred.

These experiments were extended for strain $\mathrm{H}$ to media with 0.5 and $1.0 \%$ glucose. A duplicate series of tubes was prepared containing either one of these glucose concentrations or a strip of filter-paper. In one series the $\mathrm{pH}$ was adjusted before autoclaving to $7 \cdot 0$, in the other to $7 \cdot 6$. The tubes were inoculated with cultures grown on filter paper, and incubated at $30^{\circ}$ without readjustment of the $\mathrm{pH}$. The filter-paper in the glucose-free tubes in both series was attacked after 3 days. Thus incubation at the different $\mathrm{pH}$ values did not appear to influence the development of the bacteria. In tubes with $0.5 \%$ glucose at $\mathrm{pH} 7 \cdot 0$ growth was visible after 12 days, whereas in those at $\mathrm{pH} 7 \cdot 6$ no growth was visible even after 21 days. Irrespective of the $\mathrm{pH}$, there was no growth in any of the tubes with $1.0 \%$ glucose in this period. Thus in the sugar-containing media autoclaved at $\mathrm{pH} 7 \cdot 6$ growth was impossible, whereas for the same media autoclaved at $\mathrm{pH} 7 \cdot 0$ results were comparable with those obtained in media to which separately sterilized glucose was added. 


\section{DISCUSSION}

Earlier investigations of one of us (G. F.) showed that bacteria of the 'cytophaga'-type when decomposing cellulose start by hydrolysing this polysaccharide so that finally glucose is the nutrient on which these organisms thrive. However, all available evidence suggests that in these circumstances the concentration of the glucose always remains very low. In this connexion it is instructive that our experiments leave no doubt that the bacteria in question do not grow immediately in media with relatively high glucose concentrations $(0 \cdot 5-1 \cdot 0 \%)$. Evidently the presence of glucose in these concentrations initially paralyses the normal metabolism, for if cellulose is simultaneously present it is not attacked either, during the lag period. Apparently a certain adaptation is necessary before normal metabolism becomes possible again. Then glucose is attacked preferentially, and the cellulose breakdown starts only when the greater part of the glucose has been consumed.

These results are at variance with the observations of Stanier, who reported that his strains of Sporocytophaga myxococcoides grew readily in media with a glucose concentration of $0.5 \%$ or even much higher. At present no explanation can be offered for this contrast, and we can only assume a specific difference between his strains and ours.

The authors wish to acknowledge their indebtedness to Prof. A. J. Kluyver for his interest and advice in the preparation of this paper.

\section{REFERENCES}

Fårraeus, G. (1941). Wirkung von Glukose auf die Zellulosezer-setzung einiger Cytophaga-Arten. Zbl. Bakt. (2 Abt.), 104, 264-9.

FÅratus, G. (1944). Studies in aerobic cellulose decomposition. I. The course of cellulose decomposition by Cytophaga. Ann. Agric. Coll. Sweden, 12, 1-22.

Farhraeus, G. (1946). Enzyme preparations from cellulose-decomposing bacteria. Experientia, 2, 413-15.

Fo bot. Upsalienses, 9, no. 2, 1947.

Fuller, W. H. \& Norman, A. G. (1942). Observations on some soil Cytophagas. J. Bact. 44, 256.

Fuller, W. H. \& Norman, A. G. (1943). Cellulose decomposition by aerobic mesophilic bacteria from soil. II. Biochemical studies on filter paper and cellulose preparations. J. Bact. 46, 281-8.

Harmsen, G. W. (1946). Onderzoekingen over de aerobe celluloseontleding in den grond. Dissertatie, Wageningen.

Hutchinson, H. B. \& Clayton, J. (1919). On the decomposition of cellulose by an aerobic organism (Spirochaeta cytophaga n.sp.). J. Agric. Sci. 9, 143-73.

Krzemieniewska, H. (1933). Contribution à l'étude du genre Cytophaga (Winogradsky). Arch. Mikrobiol. 4, 394-408.

Stanier, R. Y. (1940). Studies on the cytophagas. J. Bact. 40, 619-34.

Stanier, R. Y. (1942a). Are there obligate cellulose-decomposing bacteria? Soil Sci. $53,479-80$.

Stanier, R. Y. (1942b). The Cytophaga group: a contribution to the biology of Myxobacteria. Bact. Rev. 6, 143-96.

STAPP, C. \& Bontels, H. (1934). Mikrobiologische Untersuchungen über die Zersetzung von Waldstreu. Zbl. Bakt. (2. Abt.), 90, 28-66. 
Journal of General Microbiology, Vol. 3, No. 2

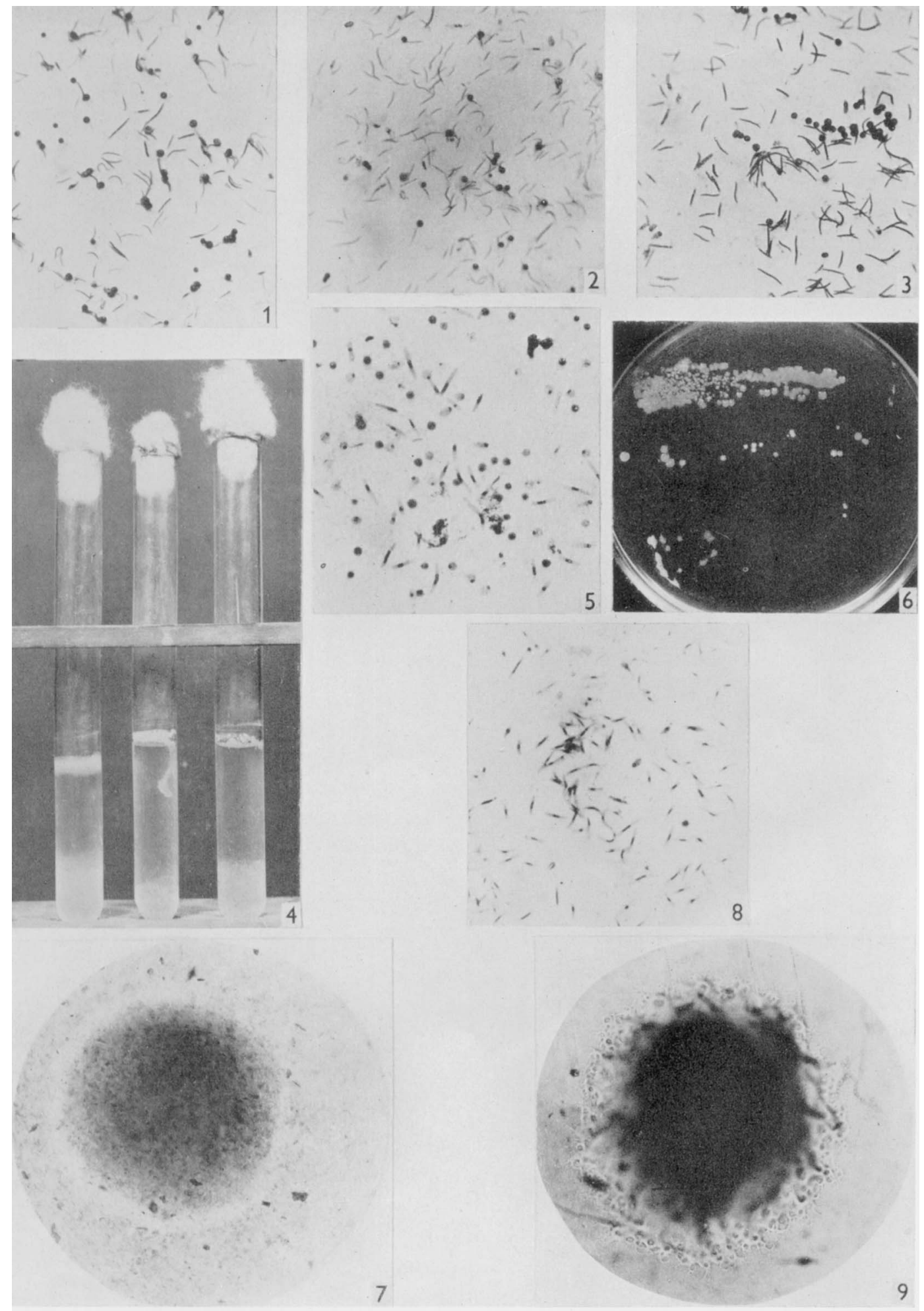

Figs. 1-9

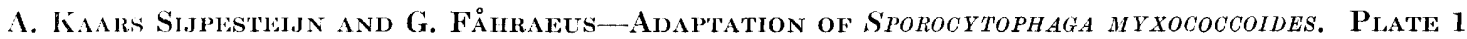





\section{EXPLANATION OF PLATE}

Fig. 1. Sporocytophaga myxococcoides, strain H. Grown on filter-paper; 5 days old; stained with Hucker's methyl violet stain for 20 min.; $\times 800$.

Fig. 2. Idem, strain W.

Fig. 3. Idem, strain $D$.

Fig. 4. Strain $\mathrm{H}$ in $1 \%$ glucose medium. Pellicle of bacteria at the surface of the medium and bacterial sediment at the bottom. Incubation period from left to right: 28,18 and 11 days.

Fig. 5. Strain $\mathbf{H}$ grown in $1 \%$ glucose medium; 5 days old; Hucker's methyl violet stain; $\times 800$.

Fig. 6. Colonies of strain $\mathrm{H}$ on $1 \%$ glucose agar. Inoculated from liquid medium with $1 \%$ glucose; 12 days old. Diameter plate $8 \mathrm{~cm}$.

Fig. 7. Colony of strain $\mathbf{H}$ on $0.1 \%$ glucose agar. Inoculated from cellulose culture; 7 days old; $\times 12$.

Fig. 8. Strain $\mathrm{H}$ grown on $0.1 \%$ glucose agar. Inoculated from cellulose culture; 8 days old; $\times 800$.

Fig. 9. Rough colony of strain $\mathrm{W}$ on $0.1 \%$ glucose agar. Inoculated from cellulose culture; 7 days old; $\times 12$.

(Received 23 August 1948) 